\title{
Fibroblasts in three dimensional matrices: cell migration and matrix remodeling
}

\author{
Sangmyung Rhee ${ }^{1}$
}

Department of Life Science

College of Natural Sciences

Chung-Ang University

Seoul 156-756, Korea

${ }^{1}$ Corresponding author: Tel, 82-2-820-5818;

Fax, 82-2-825-5206; E-mail, sangmyung.rhee@ cau.ac.kr

DOI 10.3858/emm.2009.41.12.096

Accepted 13 August 2009

Abbreviations: ECM, extracellular matrix; LPA, lysophosphatidic acid; S1P, sphingosine-1-phosphate

\begin{abstract}
Fibroblast-collagen matrix culture has facilitated the analysis of cell physiology under conditions that more closely resemble an in vivo-like environment compared to conventional 2-dimensional (2D) cell culture. Furthermore, it has led to significant progress in understanding reciprocal and adaptive interactions between fibroblasts and the collagen matrix, which occur in tissue. Recent studies on fibroblasts in 3-dimensional (3D) collagen matrices have revealed the importance of biomechanical conditions in addition to biochemical cues for cell signaling and migration. Depending on the surrounding mechanical conditions, cells utilize specific cytoskeletal proteins to adapt to their environment. More specifically, cells utilize microtubule dependent dendritic extensions to provide mechanical structure for matrix contraction under a low cell-matrix tension state, whereas cells in a high cell-matrix tension state utilize conventional acto-myosin activity for matrix remodeling. Results of collagen matrix contraction and cell migration in a 3D collagen matrix revealed that the use of appropriate growth factors led to promigratory and procontractile activity for cultured fibroblasts. Finally, the relationship between cell migration and tractional force for matrix remodeling was discussed.
\end{abstract}

Keywords: cell culture techniques; cell movement; cytoskeletal proteins; extracellular matrix; signal transduction

\section{Introduction}

The extracellular matrix (ECM) is an important component in establishing the cellular environment and provides mechanical support and a framework for cells and tissues in the body (Holmbeck and Szabova, 2006; Discher et al., 2009; Wang et al., 2009). Cells may be completely surrounded by $\mathrm{ECM}$, as is the case for chondrocytes and fibroblasts, or may attach to the ECM only at one surface, as is the case for epithelial and endothelial cells. A diverse range of binding sites for cellmatrix interaction as well as plasticity and molecular remodeling of the matrix are critical for the organization of diverse tissues, such as skin, brain and muscle, that have specific structural and mechanical properties (Rauch, 2004; Bershadsky et al., 2006; Halayko et al., 2008).

The 3 dimensional (3D) matrix-cell culture system is a useful model to analyze functional and biomechanical features of cell-matrix interactions and pathogenesis, such as wound healing (Kim, 2005; Rhee and Grinnell, 2007; Carlson et al., 2008; Lee et al., 2008). Human fibroblasts cultured in $3 \mathrm{D}$ collagen matrices encounter completely new environments relative to those experienced on the more commonly used 2D surfaces. Especially, the fibril structure of the 3D collagen matrix confers a mechanically compliant environment to the cells, which provides a reciprocal effect on cell and matrix mechanics. There are a limited number of adhesion domains accessible to the cells. In addition, the cells not only adhere through integrin-dependent 3D cell-matrix interactions, which differs from the adhesion mechanism of conventional 2D matrix-cell interactions (i.e. focal adhesion) (Cukierman et al., 2002; Petroll and Ma, 2003), but also there is a change in mechanical stability due to cell-matrix entanglement (Jiang and Grinnell, 2005). In addition, by characterizing global and local matrix remodeling induced by cell activity in the 3D collagen matrix, it would be possible to better understand the different mechanisms of cell migration in a 3D environment.

In this review, we summarize recent findings regarding fibroblast signaling in response to collagen matrix contraction and the key issues involved in understanding how the fibroblast-collagen matrix model system has been used to examine fibroblast plasticity and cell migration. 


\section{Fibroblast-mediated matrix remodeling in 3D collagen matrices}

Fibroblasts are the primary cell type responsible for collagen synthesis and the buildup of connective tissue, where the cell itself is responsible for normal tissue homeostatic processes such as tissue repair in response to injury (Hinz and Gabbiani, 2003; Desmouliere et al., 2005; Broughton et al., 2006), tissue pathology associated with fibrosis (Kisseleva and Brenner, 2008a; 2008b), and cancer progression due to stromagenesis (Beacham and Cukierman, 2005; Pavlakis et al., 2008; Castello-Cros and Cukierman, 2009). In addition, fibroblast-ECM remodeling is a pivotal design element in tissue engineering (Lutolf and Hubbell, 2005; Geutjes et al., 2006).

3D culture systems are used to mimic the natural ECM features found in vivo and, thus, they are believed to provide a tissue-like environment to cells cultured under these conditions (Kim, 2005; Pedersen and Swartz, 2005; Lee et al., 2007, 2008; Rhee and Grinnell, 2007; Yamada and Cukierman, 2007; Carlson et al., 2008). Fibroblasts in a $3 \mathrm{D}$ collagen matrix have unique morphologies ranging from dendritic to bipolar depending on matrix stiffness and tension. Moreover, fibroblasts associate with collagen fibrils, which develop reciprocal mechanical interactions with the collagen matrix. The interactions between cells and the collagen matrix result in the formation of a mechanical entanglement that transmit fibroblast force to their surrounding matrix and induces local and global matrix remodeling (contraction, compaction) (Grinnell, 1994; Jiang and Grinnell, 2005 ) to achieve tensional homoeostasis.

Matrix remodeling induced by fibroblasts depends on various factors including growth factors and the mechanical environments. To better understand the relationship between these factors and matrix remodeling, several experimental model systems were developed to examine how the cell's internal factors, such as exerted force, and extracellular factors, such as growth factors, affect matrix remodeling (Lee et al., 2003; Rhee and Grinnell, 2006; Jiang et al., 2008).

As illustrated in Figure 1, three experimental models, the floating matrix, attached matrix and restrained matrix, were developed to study the process of matrix remodeling. These models were shown to be excellent systems in regards to study the mechanism of how cells exert force to induce matrix remodeling before or after developing isometric tension (Grinnell, 2000; Lee et al., 2003). In the floating matrix model, the matrix was released after polymerization at which point the matrix started to become remodeled. Current evidence

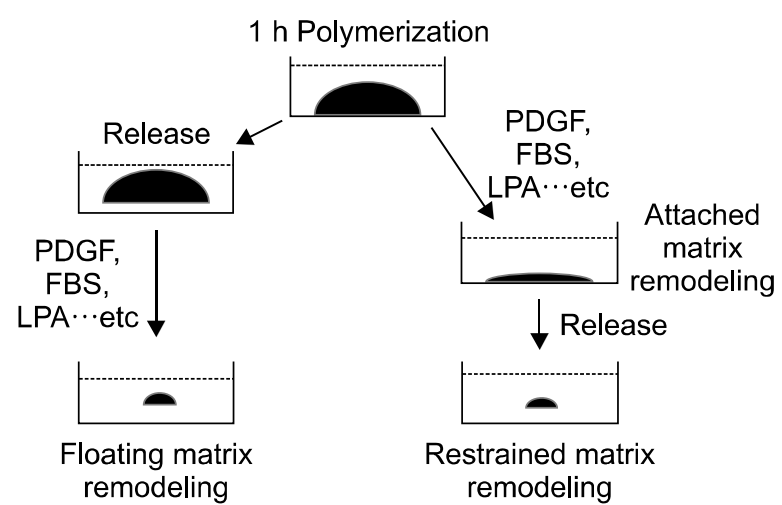

Figure 1. Comparison of in vitro collagen matrix remodeling. After one hour of collagen polymerization with fibroblasts, the matrices were either immediately released from the culture dish (floating matrix) or cultured under attached conditions (attached matrix). In the Restrained (stressed) matrix, isometric tension was developed overnight, after which the matrix was released to initiate remodeling.

suggests that floating matrix remodeling occurs as a result of the motile activity of cells, which are attempting to migrate throughout matrix (Grinnell et al., 1999; Rhee and Grinnell, 2006; Jiang et al., 2008). In contrast, cells in the attached matrix become bipolar along the line of tension and develop massive stress fibers resembling myofibroblasts (Tomasek et al., 2002; Hinz and Gabbiani, 2003). As matrix remodeling progresses in the attached matrix, the stiffness of the matrix increases and the overall mechanical properties of the matrix changes, which affects the cells' tractional activity as determined by stress fiber and focal adhesion formation (Grinnell, 2000; Shreiber et al., 2001; Tamariz and Grinnell, 2002).

In addition, a study on matrix remodeling in the restrained matrix model provided insight into understanding how an increase in the matrix mechanical loading changes the cellular mechanics. In the restrained matrix, which is under isometric tension, the mechanics controlling the cell became dependent on Rho kinase activity when LPA was used to induce remodeling (Lee et al., 2003).

\section{Signaling for matrix remodeling in fibroblast-3D collagen matrices}

Floating matrix contraction was stimulated by PDGF, LPA and serum, whereas FBS and serum were found to be better than PDGF in inducing attached matrix and restrained matrix remodeling, indicating that cells utilize different motors to stimulate matrix remodeling based on whether they are under isometric tension or not (Abe et al., 2003; Lee et al., 2003). Interestingly, PDGF-induced floating matrix contraction was required for 


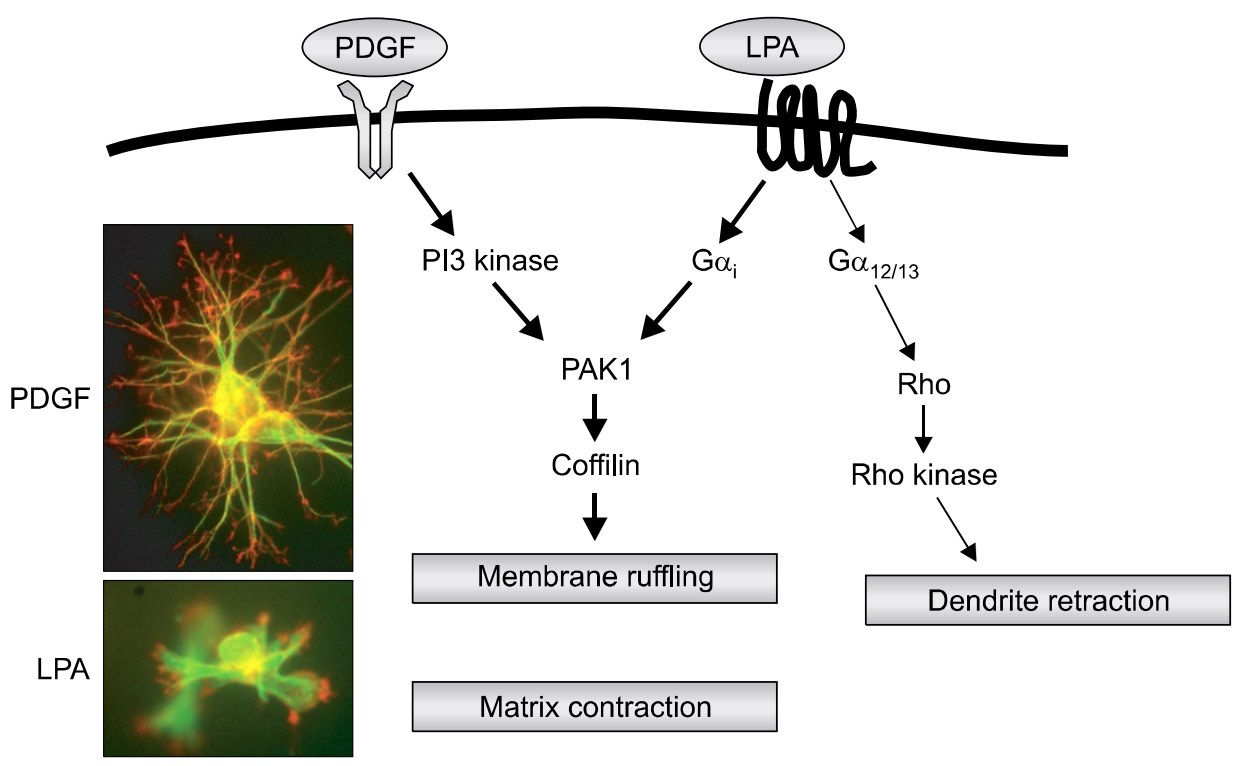

Figure 2. Membrane ruffling of fibroblast in 3D collagen matrix. The dendritic extensions of fibroblasts in $3 \mathrm{D}$ collagen matrices expanded in response to PDGF and retracted in response to LPA stimulation (Upper panel). Membrane ruffling was induced at the margin of the membrane in both PDGF- and LPAstimulated fibroblasts (Low panel), which provides mechanical activity for the floating matrix. Rho kinase and $\mathrm{mDia}$ interacted with PAK1 for PDGF and LPA-mediated matrix contraction, respectively. See Figure 3 and 10 in (Rhee and Grinnell, 2006) for more detail. phosphatidylinositol 3 kinase (PI3K) and Rho kinase activities but LPA-stimulated remodeling was independent of Rho kinase (Abe et al., 2003). By using siRNA and pharmacological inhibitors, it was demonstrated that signaling during PDGF and LPA induced floating matrix contraction was dependant on the p21-activated kinase 1 (PAK1)-coffilin signaling pathway. The different Rho effectors were coordinated with PAK1 to regulate this remodeling process, where the Rho kinase was invoIved with the antagonist PDGF and mDia1 was involved with the antagonist LPA. Moreover, the 3D collagen matrix predominately induced a cellular ruffling activity in response to both growth factors instead of lamella formation, which may play a major role in the mechanical activity of the cell during matrix remodeling (Rhee and Grinnell, 2006) (Figure 2).

However, in the case of restrained matrix remodeling, which is under isometric tension, Rho kinase-dependent myosin light chain phosphorylation was required for LPA-mediated matrix remodeling (Lee et al., 2003). Because no comparable switch occurs for PDGF-induced restrain matrix remodeling, although it has been reported that different integrins respond to specific signaling (Cooke et al., 2000), the mechanism behind PDGF-induced restrain matrix remodeling still needs to be more clearly elucidated.

\section{Fibroblast plasticity: effect of tension}

It has been extensively demonstrated that the overall morphology of fibroblasts grown in a collagen $3 \mathrm{D}$ matrix culture was determined by the physical environment of the cell (Carlson et al., 2008; Miron-Mendoza et al., 2008; Doyle et al., 2009). Indeed, it has been shown that fibroblast morphology depends on the matrix organization. In low tension states, which can be created using a floating collagen matrix, actin stress fibers were not visible in the cells, few focal adhesion phenotypes were observed and matrix biosynthesis was not observed. Interestingly, these cellular morphological traits have also been observed in resting tissue in vivo, such as during quiescence/apoptosis (Fringer and Grinnell, 2001; Graf et al., 2002; Tamariz and Grinnell, 2002; Tian et al., 2002). However, in stiff, restrained matrices, which provide a high tension state, the cell undergoes massive cytoskeletal reorganization to induce the formation of stress fibers and focal adhesions (Petroll and $\mathrm{Ma}, 2003$ ). This process then turns on matrix biosynthesis, which is analogous to cells found in active tissues such as granulation tissue and wound contraction (Kessler et al., 2001; Tomasek et al., 2002).

Changes in collagen biosynthesis and matrix stiffness by fibroblasts have also been implicated in skin aging (Papazafiri and Kletsas, 2003; Fu et al., 2005; Fisher et al., 2009). A major feature of aged skin is fragmentation of the dermal collagen matrix, which alters the mechanical properties of skin. Changes in the mechanical properties of dermal collagen can be caused by alterations in the biochemical reactions of dermal fibroblasts. Collagen synthesis in fibroblasts from aged skin was reduced and the activity of collagen-degradation enzymes was increased, which causes a tensional unbalancing of the dermal collagen ma- 
trix (Fisher et al., 2008).

\section{Microtubule function in fibroblast-3D collagen interactions}

Although it is believed that the mechanical and physical features of the extracellular environment significantly affects cell morphology (Cukierman et al., 2002; Engler et al., 2006; Vogel, 2006), it is not clear what regulatory mechanisms are involved in this process. Since fibroblasts in relaxed 3D collagen matrices have a unique morphology that contains microtubule-mediated dendritic formation, which are an indispensable factor for neurite protrusion (Lankford et al., 1990; Johnson and Stoothoff, 2004; Schaefer et al., 2008), it is reasonable to assume that microtubule-mediated signaling may be critical for determining fibroblast shape in response to different physical environments.

Figure 3 shows the differential effect of microtubules on cell spreading under 2D and 3D environments. Normally, cells cultured under 2D conditions, such as on a glass coverslip, can spread out with elongated morphologies. Interfering microtubule formation by nocodazole treatment impaired cell elongation but the extent of cell spreading without microtubules was similar to normal cells. In contrast, nocodazole treatment completely inhibi-
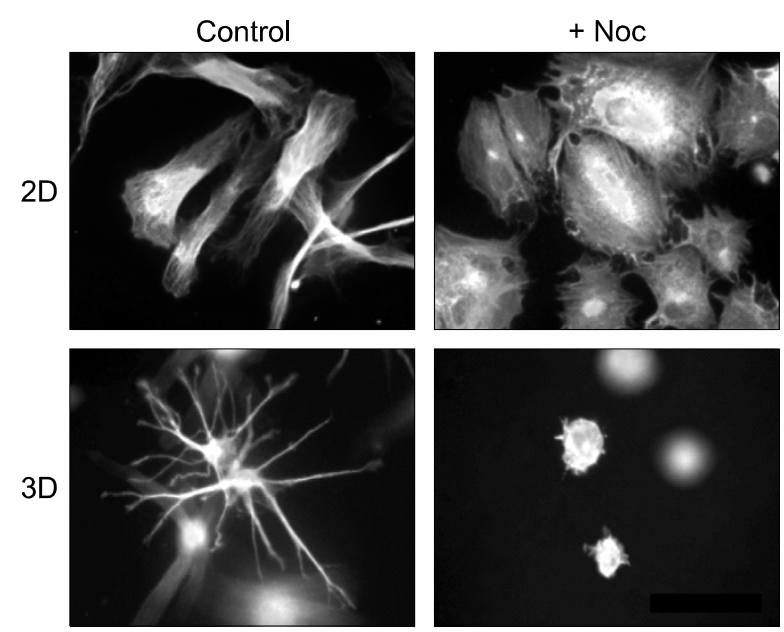

Figure 3. Different roles of microtubules in fibroblast spreading on collagen-coated coverslips compared to collagen matrices. The actin cytoskeleton was visualized and images by fluorescence microscopy. Fibroblasts cultured for $4 \mathrm{~h}$ on collagen-coated coverslips (2D) spread into an elongated, flattened morphology. Disrupting microtubules with nocodazole (+Noc) inhibited cell polarization but not spreading. Fibroblasts cultured inside of collagen matrices for $4 \mathrm{~h}$ (3D) spread by protrusion of dendritic extension. Dendritic extensions in $3 \mathrm{D}$ collagen matrices were not observed in cells that were cultured with Noc. See figure 1 in (Rhee et al., 2007) for more detail. ted cell spreading in 3D collagen matrix environments (Rhee et al., 2007). These finding indicate that the effect of microtubules on fibroblast spreading in a $3 \mathrm{D}$ collagen matrix depend on the mechanical conditions of the matrix. Although there are several differences between $2 \mathrm{D}$ and $3 \mathrm{D}$ conditions, these observations suggest that if the matrix is stiff (e.g., glass coverslip), microtubules determine cell polarity but if the matrix is soft (e.g., collagen matrix), the microtubule participates in cell spreading (Polte et al., 2004; Rhee and Grinnell, 2007; Gauthier et al., 2009).

\section{Role of the promigratory and procontractile environment in fibroblast mechanics}

Cells in a 3D matrix have distinct patterns of morphology and migration compared with those on a 2D planar surface (Friedl and Brocker, 2000; Friedl, 2004; Zaman et al., 2006). Especially, when fibroblasts are on a 2D planar surface, the tractional force against a stiff substrate results in cell migration. However, when cells are in a 3D collagen matrix, the tractional force can be utilized as a mechanical inducer for matrix remodeling both locally and globally. Therefore, understanding the molecular mechanism of fibroblast migration in a 3D matrix can be important for understanding various pathological diseases and fibrotic diseases including fibrosis and cancer metastasis (Desmouliere et al., 2004; Abraham et al., 2007; Kenny et al., 2007).

It was originally shown that fibroblasts can migrate out of tissue explants embedded in collagen matrices (Sawhney and Howard, 2002) and also migrate from contracted cells containing collagen matrices into fibronectin-fibrin matrices (Greiling and Clark, 1997; Clark et al., 2003; Lin et al., 2005). Researchers have recently taking advantage of these observations to develop the nested collagen matrix system (Grinnell et al., 2006). In this system, dermal equivalents, which were prepared from precontracted floating collagen matrices, were embedded in cell-free collagen matrices. Unlike the process of durotaxis, where cell migration occurs under a gradient of matrix rigidity (Lo et al., 2000), fibroblast migration can occur from a hard matrix to a soft matrix in a nest collagen matrix along the chemoattractants gradient, which is a distinct migration pattern in the context of the 3D matrix (Grinnell et al., 2006; Miron-Mendoza et al., 2008).

Several interesting findings in relation to fibroblast migration were obtained using the nested matrix. Initially, there was an 8-16 h lag phase before migration began, which has never been 
observed on a 2D planner surface. However, it has not yet been clearly determined why the cell needs such a long lag phase before migration starts. One plausible explanation is that a certain period of time is required for the cells to overcome the difference in matrix stiffness between the inner and outer matrix. Another possible reason is that the cells need to develop biosynthetic features to simulate a resting dermis, since almost all signaling would already have been turned off in a dermal equivalent (Grinnell et al., 2003). In addition, by examining fibroblast migration in the nested matrix, collagen fibril flow was shown to occur from the outer matrix toward the inner matrix prior to fibroblast migration (Miron-Mendoza et al., 2008). Furthermore, this method can be used as a useful tool for screening of oncogenic transformation (Menezes et al., 2008).

Experiments on matrix contraction and nested matrix cell migration have provided a better understanding of the molecular mechanisms responsible for fibroblast activity in the presence of different growth factors (Jiang et al., 2008). In the process of wound healing, especially, fibroblasts might encounter various growth factors that regulate cell migration and matrix contraction. During wound repair, fibroblasts migrate into the wound area and consequently develop mechanical stress and matrix contraction (Fu et al., 2005; Davidson, 2007). Therefore, it is important to understand the molecular mechanisms by which growth factors participate in distinct cellular processes in wound environments. Jiang et al tested several purified agonists and serum for matrix contraction and cell migration in a nested collagen matrix. In this study, they demonstrated that PDGF was the only growth factor capable of inducing cell migration in a nested matrix. However, PDGF displayed weak activity under stress matrix contraction. In contrast, lipid variant agonists including serum, LPA and S1P were found to promote stress-released matrix contraction but not cell migration. Moreover, S1P worked as a master regulator for balancing the promigratory and procontractile conditions (Jiang et al., 2008).

\section{Conclusion}

The development of 3D model systems in cell biology has recently emerged as an important tool to study cellular behavior since cell physiology is quite different in a 2D versus $3 \mathrm{D}$ environment. In addition, 3D experiments allow one to capture the complex 3D tissue physiology under in vitro conditions (Grinnell, 2003; Griffith and Swartz,
2006; Krause et al., 2008; Kuo and Tuan, 2008). Furthermore, 3D model systems provide useful tools for understanding the mechanobiology of how individual cells sense and generate mechanical force in response to their surrounding environment (Pedersen and Swartz, 2005).

Thus far, most studies on cell mechanics have been carried out in high tension and 2D environments, which is presented as a one-way signaling event; however, the fibroblast-3D matrix system permits cells to have reciprocal, two-way interactions between the matrix and cells. Cells interacting under low-tensional matrix conditions can convert the matrix mechanics from soft to stiff in response to growth factor stimulation, which can eventually promote high-tensional interactions between cells. During these events, all the dynamic changes in the cell-matrix interactions under different mechanical environments greatly influence cell signaling and behavior.

In addition to the mechanical environment, the presence of different growth factors can also influence cell behavior in a 3D matrix. In this regard, differences in cell signaling, cytoskeleton modulation and integrin-focal dynamics during promigratory processes, such as in the presence of PDGF and procontractile processes, such as in the presence of serum and FBS, are critical for cell mechanics and cell-matrix interaction. Therefore, the combination of growth factor signaling and changes in the mechanical environment by cell-matrix interactions provides a new concept for understanding the relationship between cellular behavior and matrix mechanics (so called four quadrants) (Rhee and Grinnell, 2007).

Finally, cellular mechanics in different biomechanical and growth factor environments constitutes a new research field that still needs to be developed. For example, pathological diseases such as cancer progression and wound repair as well as aging require growth factor stimulation, which can alter the mechanical environment and change the cellmatrix interaction. It has been accepted that growth factor signaling changes the cell behaviors and dynamics; but, the effect of growth factors on mechanical changes in a matrix environment cannot be credibly established when experiments are carried out in 2D culture systems. However, these limitations can be overcome using a 3D culture system. 3D culture systems allow researchers to decouple multiple molecular and dynamic interactions between cells and a surrounding matrix, since the system acts as an in vivo mimic of biological tissue. Further studies exploring in greater detail the process of in vitro remodeling using fibroblast3D matrix culture systems will be highly valuable in 
resolving some of the challenges currently facing the field of 3D biology.

\section{Acknoledgements}

I thank Dr. Frederick Grinnell (University of Texas Southwestern Medical Center, Dallas, TX) for critical reading and helpful comments. This Research was supported by the Chung-Ang University Research Grants in 2008.

\section{References}

Abe M, Ho CH, Kamm KE, Grinnell F. Different molecular motors mediate platelet-derived growth factor and lysophosphatidic acid-stimulated floating collagen matrix contraction. J Biol Chem 2003;278:47707-12

Abraham DJ, Eckes B, Rajkumar V, Krieg T. New developments in fibroblast and myofibroblast biology: implications for fibrosis and scleroderma. Curr Rheumatol Rep 2007;9:136-43

Beacham DA, Cukierman E. Stromagenesis: the changing face of fibroblastic microenvironments during tumor progression. Semin Cancer Biol 2005;15:329-41

Bershadsky AD, Ballestrem C, Carramusa L, Zilberman $Y$, Gilquin B, Khochbin S, Alexandrova AY, Verkhovsky AB, Shemesh T, Kozlov MM. Assembly and mechanosensory function of focal adhesions: experiments and models. Eur $\mathrm{J}$ Cell Biol 2006;85:165-73

Broughton G, 2nd, Janis JE, Attinger CE. The basic science of wound healing. Plast Reconstr Surg 2006;117:12S-34S

Carlson MW, Alt-Holland A, Egles C, Garlick JA. Threedimensional tissue models of normal and diseased skin. Curr Protoc Cell Biol 2008;19:Unit 199

Castello-Cros R, Cukierman E. Stromagenesis During Tumorigenesis: Characterization of Tumor-associated Fibroblasts and Stroma-derived 3D Matrices. Methods Mol Biol 2009;522:1-31

Clark RA, An JQ, Greiling D, Khan A, Schwarzbauer JE. Fibroblast migration on fibronectin requires three distinct functional domains. J Invest Dermatol 2003;121:695-705

Cooke ME, Sakai T, Mosher DF. Contraction of collagen matrices mediated by alpha2beta1A and alpha(v)beta3 integrins. J Cell Sci 2000;113(Pt 13):2375-83

Cukierman E, Pankov R, Yamada KM. Cell interactions with three-dimensional matrices. Curr Opin Cell Biol 2002;14: 633-9

Davidson JM. Growth factors: the promise and the problems. Int J Low Extrem Wounds 2007;6:8-10

Desmouliere A, Guyot C, Gabbiani G. The stroma reaction myofibroblast: a key player in the control of tumor cell behavior. Int J Dev Biol 2004;48:509-17

Desmouliere A, Chaponnier C, Gabbiani G. Tissue repair, contraction, and the myofibroblast. Wound Repair Regen 2005;13:7-12
Discher D, Dong C, Fredberg JJ, Guilak F, Ingber D, Janmey $\mathrm{P}$, Kamm RD, Schmid-Schonbein GW, Weinbaum S. Biomechanics: Cell Research and Applications for the Next Decade. Ann Biomed Eng 2009;37:847-59

Doyle AD, Wang FW, Matsumoto K, Yamada KM. Onedimensional topography underlies three-dimensional fibrillar cell migration. J Cell Biol 2009;184:481-90

Engler AJ, Sen S, Sweeney HL, Discher DE. Matrix elasticity directs stem cell lineage specification. Cell 2006;126:677-89

Fisher GJ, Varani J, Voorhees JJ. Looking older: fibroblast collapse and therapeutic implications. Arch Dermatol 2008; 144:666-72

Fisher GJ, Quan T, Purohit T, Shao Y, Cho MK, He T, Varani J, Kang S, Voorhees JJ. Collagen fragmentation promotes oxidative stress and elevates matrix metalloproteinase- 1 in fibroblasts in aged human skin. Am J Pathol 2009;174:10114

Friedl P. Prespecification and plasticity: shifting mechanisms of cell migration. Curr Opin Cell Biol 2004;16:14-23

Friedl P, Brocker EB. The biology of cell locomotion within three-dimensional extracellular matrix. Cell Mol Life Sci 2000;57:41-64

Fringer J, Grinnell F. Fibroblast quiescence in floating or released collagen matrices: contribution of the ERK signaling pathway and actin cytoskeletal organization. J Biol Chem 2001;276:31047-52

Fu X, Li X, Cheng B, Chen W, Sheng Z. Engineered growth factors and cutaneous wound healing: success and possible questions in the past 10 years. Wound Repair Regen 2005;13:122-30

Gauthier NC, Rossier OM, Mathur A, Hone JC, Sheetz MP. Plasma Membrane Area Increases with Spread Area by Exocytosis of a GPI Anchored Protein Compartment. Mol Biol Cell 2009;20:3261-72

Geutjes PJ, Daamen WF, Buma P, Feitz WF, Faraj KA, van Kuppevelt TH. From molecules to matrix: construction and evaluation of molecularly defined bioscaffolds. Adv Exp Med Biol 2006;585:279-95

Graf R, Freyberg M, Kaiser D, Friedl P. Mechanosensitive induction of apoptosis in fibroblasts is regulated by thrombospondin-1 and integrin associated protein (CD47). Apoptosis 2002;7:493-8

Greiling D, Clark RA. Fibronectin provides a conduit for fibroblast transmigration from collagenous stroma into fibrin clot provisional matrix. J Cell Sci 1997;110(Pt 7):861-70

Griffith LG, Swartz MA. Capturing complex 3D tissue physiology in vitro. Nat Rev Mol Cell Biol 2006;7:211-24

Grinnell F. Fibroblasts, myofibroblasts, and wound contraction. J Cell Biol 1994;124:401-4

Grinnell F. Fibroblast-collagen-matrix contraction: growthfactor signalling and mechanical loading. Trends Cell Biol 2000;10:362-5

Grinnell F. Fibroblast biology in three-dimensional collagen matrices. Trends Cell Biol 2003;13:264-9 
Grinnell F, Ho CH, Lin YC, Skuta G. Differences in the regulation of fibroblast contraction of floating versus stressed collagen matrices. J Biol Chem 1999;274:918-23

Grinnell F, Ho CH, Tamariz E, Lee DJ, Skuta G. Dendritic fibroblasts in three-dimensional collagen matrices. Mol Biol Cell 2003;14:384-95

Grinnell F, Rocha LB, lucu C, Rhee S, Jiang H. Nested collagen matrices: a new model to study migration of human fibroblast populations in three dimensions. Exp Cell Res 2006;312:86-94

Halayko AJ, Tran T, Gosens R. Phenotype and functional plasticity of airway smooth muscle: role of caveolae and caveolins. Proc Am Thorac Soc 2008;5:80-8

Hinz B, Gabbiani G. Mechanisms of force generation and transmission by myofibroblasts. Curr Opin Biotechnol 2003; $14: 538-46$

Holmbeck K, Szabova L. Aspects of extracellular matrix remodeling in development and disease. Birth Defects Res C Embryo Today 2006;78:11-23

Jiang $H$, Grinnell F. Cell-matrix entanglement and mechanical anchorage of fibroblasts in three-dimensional collagen matrices. Mol Biol Cell 2005;16:5070-6

Jiang $\mathrm{H}$, Rhee $\mathrm{S}, \mathrm{Ho} \mathrm{CH}$, Grinnell F. Distinguishing fibroblast promigratory and procontractile growth factor environments in 3-D collagen matrices. FASEB J 2008;22:2151-60

Johnson GV, Stoothoff WH. Tau phosphorylation in neuronal cell function and dysfunction. J Cell Sci 2004;117:5721-9

Kenny HA, Krausz T, Yamada SD, Lengyel E. Use of a novel $3 \mathrm{D}$ culture model to elucidate the role of mesothelial cells, fibroblasts and extra-cellular matrices on adhesion and invasion of ovarian cancer cells to the omentum. Int J Cancer 2007;121:1463-72

Kessler D, Dethlefsen S, Haase I, Plomann M, Hirche F, Krieg T, Eckes B. Fibroblasts in mechanically stressed collagen lattices assume a "synthetic" phenotype. J Biol Chem 2001;276:36575-85

Kim JB. Three-dimensional tissue culture models in cancer biology. Semin Cancer Biol 2005;15:365-77

Kisseleva T, Brenner DA. Fibrogenesis of parenchymal organs. Proc Am Thorac Soc 2008a;5:338-42

Kisseleva T, Brenner DA. Mechanisms of fibrogenesis. Exp Biol Med (Maywood) 2008b;233:109-22

Krause S, Maffini MV, Soto AM, Sonnenschein C. ANovel 3D In Vitro Culture Model to Study Stromal-Epithelial Interactions in the Mammary Gland. Tissue Eng Part C Methods 2008; $14: 261-71$

Kuo CK, Tuan RS. Mechanoactive tenogenic differentiation of human mesenchymal stem cells. Tissue Eng Part A 2008;14:1615-27

Lankford K, Cypher C, Letourneau P. Nerve growth cone motility. Curr Opin Cell Biol 1990;2:80-5

Lee DJ, Ho CH, Grinnell F. LPA-stimulated fibroblast contraction of floating collagen matrices does not require Rho kinase activity or retraction of fibroblast extensions. Exp
Cell Res 2003;289:86-94

Lee GY, Kenny PA, Lee EH, Bissell MJ. Three-dimensional culture models of normal and malignant breast epithelial cells. Nat Methods 2007;4:359-65

Lee J, Cuddihy MJ, Kotov NA. Three-dimensional cell culture matrices: state of the art. Tissue Eng Part B Rev 2008;14:61-86

Lin F, Ren XD, Doris G, Clark RA. Three-dimensional migration of human adult dermal fibroblasts from collagen lattices into fibrin/fibronectin gels requires syndecan-4 proteoglycan. J Invest Dermatol 2005;124:906-13

Lo CM, Wang HB, Dembo M, Wang YL. Cell movement is guided by the rigidity of the substrate. Biophys $\mathrm{J}$ 2000;79:144-52

Lutolf MP, Hubbell JA. Synthetic biomaterials as instructive extracellular microenvironments for morphogenesis in tissue engineering. Nat Biotechnol 2005;23:47-55

Menezes GC, Miron-Mendoza M, Ho CH, Jiang H, Grinnell F. Oncogenic Ras-transformed human fibroblasts exhibit differential changes in contraction and migration in 3D collagen matrices. Exp Cell Res 2008;314:3081-91

Miron-Mendoza M, Seemann J, Grinnell F. Collagen Fibril Flow and Tissue Translocation Coupled to Fibroblast Migration in 3D Collagen Matrices. Mol Biol Cell 2008;19:2051-8

Papazafiri P, Kletsas D. Developmental and age-related alterations of calcium homeostasis in human fibroblasts. Exp Gerontol 2003;38:307-11

Pavlakis K, Messini I, Vrekoussis T, Yiannou P, Keramopoullos D, Louvrou N, Liakakos T, Stathopoulos EN. The assessment of angiogenesis and fibroblastic stromagenesis in hyperplastic and pre-invasive breast lesions. BMC Cancer 2008;8:88

Pedersen JA, Swartz MA. Mechanobiology in the third dimension. Ann Biomed Eng 2005;33:1469-90

Petroll WM, Ma L. Direct, dynamic assessment of cell-matrix interactions inside fibrillar collagen lattices. Cell Motil Cytoskeleton 2003;55:254-64

Polte TR, Eichler GS, Wang N, Ingber DE. Extracellular matrix controls myosin light chain phosphorylation and cell contractility through modulation of cell shape and cytoskeletal prestress. Am J Physiol Cell Physiol 2004;286: C518-28

Rauch U. Extracellular matrix components associated with remodeling processes in brain. Cell Mol Life Sci 2004; 61:2031-45

Rhee S, Grinnell F. P21-activated kinase 1: convergence point in PDGF- and LPA-stimulated collagen matrix contraction by human fibroblasts. J Cell Biol 2006;172:423-32

Rhee S, Grinnell F. Fibroblast mechanics in 3D collagen matrices. Adv Drug Deliv Rev 2007;59:1299-305

Rhee $\mathrm{S}$, Jiang $\mathrm{H}, \mathrm{Ho} \mathrm{CH}$, Grinnell F. Microtubule function in fibroblast spreading is modulated according to the tension state of cell-matrix interactions. Proc Natl Acad Sci USA 2007;104:5425-30 
Sawhney RK, Howard J. Slow local movements of collagen fibers by fibroblasts drive the rapid global self-organization of collagen gels. J Cell Biol 2002;157:1083-91

Schaefer AW, Schoonderwoert VT, Ji L, Mederios N, Danuser G, Forscher P. Coordination of actin filament and microtubule dynamics during neurite outgrowth. Dev Cell 2008;15:146-62

Shreiber DI, Enever PA, Tranquillo RT. Effects of pdgf-bb on rat dermal fibroblast behavior in mechanically stressed and unstressed collagen and fibrin gels. Exp Cell Res 2001; 266:155-66

Tamariz E, Grinnell F. Modulation of fibroblast morphology and adhesion during collagen matrix remodeling. Mol Biol Cell 2002;13:3915-29

Tian B, Lessan K, Kahm J, Kleidon J, Henke C. beta 1 integrin regulates fibroblast viability during collagen matrix contraction through a phosphatidylinositol 3-kinase/Akt/protein kinase B signaling pathway. J Biol Chem 2002;277:24667-75

Tomasek JJ, Gabbiani G, Hinz B, Chaponnier C, Brown RA. Myofibroblasts and mechano-regulation of connective tissue remodelling. Nat Rev Mol Cell Biol 2002;3:349-63

Vogel V. Mechanotransduction involving multimodular proteins: converting force into biochemical signals. Annu Rev Biophys Biomol Struct 2006;35:459-88

Wang N, Tytell JD, Ingber DE. Mechanotransduction at a distance: mechanically coupling the extracellular matrix with the nucleus. Nat Rev Mol Cell Biol 2009;10:75-82

Yamada KM, Cukierman E. Modeling tissue morphogenesis and cancer in 3D. Cell 2007;130:601-10

Zaman MH, Trapani LM, Sieminski AL, Mackellar D, Gong $\mathrm{H}$, Kamm RD, Wells A, Lauffenburger DA, Matsudaira P. Migration of tumor cells in 3D matrices is governed by matrix stiffness along with cell-matrix adhesion and proteolysis. Proc Natl Acad Sci USA 2006;103:10889-94 